\title{
Palinologia de Amaranthaceae e Araliaceae nativas em fragmentos florestais remanescentes da região noroeste do Estado de São Paulo
}

\author{
Lorrayne Albernaz Domingues Camilo Landi ${ }^{1}$ e Eduardo Custódio Gasparino ${ }^{1,2}$
}

Recebido: 9.09.2017; aceito: 20.12.2017

\begin{abstract}
Palynology of native Amaranthaceae and Araliaceae in forest fragments of the Northwest of São Paulo State). We investigated the pollen morphology of two native species of Amaranthaceae and three of Araliaceae from forest fragments in order to contribute with the palynology, taxonomy, and conservation of degraded areas. The pollen grains were acetolyzed, measured, qualitatively described and photographed using light and scanning electron microscopy. Quantitative data were analyzed by descriptive statistics. The pollen grains of Amaranthaceae are monads, small, apolar, spherical, pantoporate, and with metareticulate exine. For Araliaceae, the pollen grains are monads, small to medium, isopolar, subcircular to subtriangular, oblato-spheroidal to subprolate, 3-colporate, sometimes with vestibulum, lalongate endoaperture and microreticulate exine. The pollen data confirmed Amaranthaceae as a stenopalynous family. On the other hand, the species of Araliaceae studied characteristically present pollen grains apertures that can be used to differentiate them.
\end{abstract}

Keywords: Brazil, pollen grains, pollen morphology, stenopalynous

RESUMO - (Palinologia de Amaranthaceae e Araliaceae nativas em fragmentos florestais remanescentes da Região Noroeste do Estado de São Paulo). Foram investigados os grãos de pólen de duas espécies de Amaranthaceae e três espécies de Araliaceae ocorrentes em fragmentos florestais remanescentes, contribuindo dessa forma com a palinologia, taxonomia e conservação de áreas degradadas. Os grãos de pólen foram acetolisados, medidos, descritos qualitativamente e fotografados sob microscopia de luz e de varredura. Os dados quantitativos foram analisados por estatística descritiva. Os grãos de pólen das espécies de Amaranthaceae são mônades, pequenos, apolares, esféricos, pantoporados com exina metarreticulada. Os das espécies de Araliaceae são mônades, pequenos a médios, isopolares, âmbito subcircular a subtriangular, oblatoesferoidais a subprolatos, 3-colporados, algumas vezes com vestíbulo, endoaberturas lalongadas e exina microrreticulada. Os dados obtidos confirmam o caráter estenopolínico de Amaranthaceae, no entanto as espécies de Araliaceae apresentam características de abertura dos seus grãos de pólen que podem ser usadas para diferenciá-las.

Palavras-chave: Brasil, estenopolínico, grãos de pólen, morfologia polínica

\section{Introdução}

A formação vegetal da região noroeste do Estado de São Paulo é composta essencialmente por Floresta Estacional Semidecidual e Cerrado, e atualmente é um exemplo claro da devastação vegetal, ocorrida principalmente por substituição de sua cobertura original, por pastagens, culturas diversas ou áreas urbanas (Necchi 2012). Segundo Maury (2002), os estudos realizados em áreas prioritárias para a conservação, constituídas por remanescentes de Floresta Estacional Semidecidual, demonstram ser consideradas como "de provável importância biológica, mas insuficientemente conhecida".
O maior impacto da fragmentação florestal é a perda da biodiversidade regional, e quanto mais fragmentadas e perturbadas são as paisagens, maiores são os desafios para conservação destas áreas (Viana 1995). Dessa forma, estudos florísticos realizados em regiões fragmentadas, incluindo estudos de flora polínica, auxiliam na identificação de espécies remanescentes, ampliando o conhecimento dos táxons de áreas degradadas.

As famílias Amaranthaceae e Araliaceae foram catalogadas pelo projeto "Fauna e Flora de Fragmentos Florestais remanescentes da Região Noroeste do Estado de São Paulo" (Necchi 2012), cujo intuito foi preencher

1. Universidade Estadual Paulista, Faculdade de Ciências Agrárias e Veterinárias de Jaboticabal, Departamento de Biologia aplicada à Agropecuária, Via de acesso Prof. Paulo Donato Castellane s/n, 14.884-900 Jaboticabal, SP, Brasil

2. Autor para correspondência: ecgasparino@fcav.unesp.br 
a necessidade do conhecimento sobre a biodiversidade da Região Noroeste do Estado de São Paulo, determinando a riqueza e a diversidade de espécies e analisando os possíveis efeitos da fragmentação nessas comunidades. Estas famílias estão presentes em 17 fragmentos dos 18 fragmentos florestais amostrados, sendo Amaranthaceae representada por duas espécies, Hebanthe eriantha (Poir.) Pedersen e Pfaffia glomerata (Spreng.) Pedersen, e Araliaceae representada por quatro espécies, Aralia excelsa (Griseb.) J.Wen, Dendropanax cuneatus (DC.) Decne. \& Planch., Schefflera morototoni (Aubl.) Maguire, Steyerm \& Frodin e Schefflera vinosa (Cham. \& Schltdl.) Frodin \& Fiaschi. Segundo Marchioretto et al. (2015) e Fiaschi (2015) as espécies brasileiras de Amaranthaceae e Araliaceae estão localizadas em sua maioria na região sudeste do país, localizadas preferencialmente no Cerrado e Mata Atlântica.

De acordo com o APG IV (2016), Amaranthaceae está inserida em Caryophyllales, juntamente com 37 famílias. Possui cerca de 2.500 espécies, distribuídas entre 130 gêneros anteriormente reconhecidos em Chenopodiaceae e 79 gêneros de Amaranthaceae sensu stricto. No Brasil, a família possui 158 espécies, distribuídas em 27 gêneros, e destas, 74 espécies são consideradas endêmicas (Marchioretto et al. 2015). Araliaceae está posicionada em Apiales juntamente com mais seis famílias, apresenta cerca de 1.450 espécies, distribuídas em 43 gêneros (APG IV 2016). São descritos para o território brasileiro cinco gêneros de Araliaceae, os quais compreendem 93 espécies, sendo 56 endêmicas (Fiaschi 2015).

Dentre os trabalhos que analisaram as características polínicas de espécies de Amaranthaceae destacam-se: Erdtman (1952), Salgado-Labouriau (1973), Barth et al. (1976), Melhem \& Cruz (1979), Nowicke \& Skvarla (1979), Robertson (1981), Eliasson (1988), Borsch (1995, 1998), Telleria (1995), Borsch \& Pedersen (1997), Franssen et al. (2001), Perveen \& Qaiser (2002), Melhem et al. (2003), Evaldt et al. (2009), Bastos et al. (2010), Chin \& Lim (2011), Angelini et al. (2014), Magalhães-e-Silva et al. (2016) e Sanchez-del-Pino et al. (2016). Para as espécies de Araliaceae, dados palinológicos foram encontados em: Erdtman (1952), Chung \& Huang (1972), SalgadoLabouriau (1973), Tseng \& Shoup (1978), Tseng et al. (1983), Van Helvoort \& Punt (1984), Melhem \& Bissa (1985), Shang \& Callen (1988), Pire (1989), Henwood (1991), Wen \& Nowicke (1999), Melhem et al. (2003), e Fiaschi et al. (2008, 2010). Estes estudos relatam a morfologia polínica destas famílias, e grande parte dos dados apresentados nestes contribuem para melhor delimitação de espécies, gêneros e até mesmo subfamílias, fornecendo dados morfológicos que servirão de base para o entendimento das relações entre táxons. Desta forma, o objetivo deste trabalho foi caracterizar a morfologia polínica das espécies de Amaranthaceae e Araliaceae ocorrentes em regiões degradadas, fornecendo subsídios para o melhor conhecimento morfológico dos táxons, auxiliando assim na sua conservação.

\section{Material e métodos}

Foram estudados os grãos de pólen de cinco espécies sendo duas espécies de Amaranthaceae (Hebanthe eriantha (Poir.) Pedersen e Pfaffia glomerata (Spreng.) Pedersen) e três espécies de Araliaceae (Aralia excelsa (Griseb.) J.Wen, Dendropanax cuneatus (DC.) Decne. \& Planch. e Schefflera vinosa (Cham. \& Schltdl.) Frodin \& Fiaschi). As espécies apresentam-se distribuídas em fragmentos amostrados pelo projeto "Fauna e Flora de Fragmentos Florestais remanescentes da Região Noroeste do Estado de São Paulo" (Necchi 2012) e foram citadas por Ranga et al. (2012) como nativas destas áreas. A espécie Schefflera morototoni (Aubl.) Maguire, Steyerm \& Frodin, também citada por Ranga et al. (2012), não foi analisada por falta de material polínico. Os materiais utilizados para as preparações polínicas foram obtidos a partir de exsicatas do herbário CESJ (Herbário Leopoldo Krieger da Universidade Federal de Juiz de Fora) e do herbário SJRP (Departamento de Zoologia e Botânica, Universidade Estadual Paulista, São José do Rio Preto). Para cada espécie estudada, foram analisadas até três espécimes para confirmação dos dados obtidos; destes, um de cada foi utilizado como material padrão para caracterização da morfologia polínica, medidas e ilustrações. A escolha do espécime padrão decorreu da melhor resistência dos grãos de pólen ao processo de acetólise, os demais espécimes foram considerados materiais de comparação. Os espécimes estudados estão listados a seguir, em ordem alfabética e os espécimes-padrão indicados por um asterisco.

Material examinado: Amaranthaceae - Hebanthe eriantha (Poir.) Pedersen: BRASIL. MINAS GeraIs: Leopoldina, 20-IX-1986, L. Krieger, 21300 (CESJ). SÃo Paulo: Botucatu, Fazenda Edgardia, 19-IX2007, C.J. Campos, M.R. Osti \& M.B. Fiamengui 6 (SJRP); Campinas, Distrito de Sousa, Ribeirão Cachoeiras, 24-IX-1996, K. Santos, s/n (SJRP30112)*. 
Pfaffia glomerata (Spreng.) Pedersen: BRASIL. Minas Gerais: Santa Rita de Jacutinga, 24-I-1971, P. L. Krieger, 9874 (CESJ). ParanÁ: Ponta Grossa, Uraia, P. L. Krieger, 14361 (CESJ)*. São PaUlo: São José do Rio Preto, 30-VIII-2002, J.B. Rezende et al., s/n (SJRP27885). Araliaceae - Aralia excelsa (Griseb.) J.Wen: BRASIL. São PAUlo: Jaboticabal, Fazenda Santa Isabel, 19-III-1994, E.A. Rodrigues, 201 (JABU)*. Dendropanax cuneatus (DC.) Decne. \& Planch: BRASIL. São PAulo: Matão, Fazenda Cambuhy, 27-VI-2007, V. Stranghetti, N.T. Ranga \& A. Rezende, 932 (SJRP)*; Município de Paulo de Faria, Estação Ecológica de Paulo de Faria, 29-VI-2001, F. Tomasetto \& A.A. Rezende 165 (SJRP); Novo Horizonte, Fazenda Serrinha, 30-V-2007, V. Stranghetti, N.T. Ranga \& A. Rezende 934 (SJRP). Schefflera vinosa (Cham. \& Schltdl.) Frodin \& Fiaschi: BRASIL. São PAULO: Presidente Bernardes, Região do Pontal Pranapanema, 10-III-1996, M.S. Pietrobom 3171 (SJRP); Sales, Fazenda Águas Claras, 24-V-2008, V. Stranghetti, N.T. Ranga \& A. Rezende 941 (SJRP); Sales, Fazenda Águas Claras, 24-V-2008, V. Stranghetti, N.T. Ranga \& A. Rezende 942 (SJRP)*.

Os grãos de pólen foram analisados em microscopia óptica e microscopia eletrônica de varredura. Para a microscopia óptica os grãos de pólen foram preparados pelo método de acetólise de Erdtman (1960), acrescidas das modificações citadas por Melhem et al. (2003), e medidos aleatoriamente dentro do prazo máximo de uma semana (Melhem \& Matos 1972). Para os diâmetros, foram realizadas medidas em 25 grãos de pólen, distribuídos em pelo menos quatro lâminas, visando uma uniformidade da amostra (Melhem \& Matos 1972, Salgado-Labouriau 1973). Para outros caracteres como diâmetros dos poros, comprimento e largura dos cólporos, medidas das endoaberturas, apocolpo e espessura das camadas da exina, bem como os diâmetros dos materiais de comparação, foram feitas 10 medidas. As lâminas foram incorporadas na Palinoteca do Laboratório de Morfologia Vegetal e Palinologia do Departamento de Biologia aplicada a Agropecuária, Universidade Estadual Paulista, Unesp, Jaboticabal, SP, Brasil.

Dos dados quantitativos obtidos, foram calculados a média aritmética ( $\mathrm{x}$ ), o desvio padrão da média (sx), o desvio padrão da amostra (s), o coeficiente de variabilidade $(\mathrm{CV})$ e o intervalo de confiança a $95 \%$ (IC), para as medidas com $n=25$. Para as demais medidas calculou-se apenas a média aritmética.

A análise em microscopia eletrônica de varredura seguiu o proposto por Melhem et al. (2003) para grãos de pólen não acetolisados.
Para o registro das imagens, fotomicrografias dos grãos de pólen em microscopia óptica foram realizadas com o auxílio de um microscópio óptico e uma câmera de vídeo acoplada (Leica IM50, Bel Photonics) a um microcomputador. Já em microscopia eletrônica de varredura, os grãos de pólen foram fotografados digitalmente no microscópio eletrônico de varredura (MEV) JEOL modelo JSM5410. A partir destas imagens foram elaboradas pranchas para ilustração dos grãos de pólen analisados.

As descrições polínicas e a terminologia foram baseadas respectivamente nos glossários de Barth \& Melhem (1988) e Punt et al. (2007), o índice de área polar e o índice de largura do colpo foram descritos de acordo com Faegri \& Iversen (1966) e Gasparino et al. (2013) respectivamente. O cálculo do número de poros em Amarathaceae seguiu Angelini et al. (2014).

\section{Resultados}

Família Amaranthaceae - Espécies analisadas: Hebanthe eriantha (Poir.) Pedersen. (figuras 1-5; tabelas 1-4). Pfaffia glomerata (Spreng.) Pedersen (figuras 6-9; tabelas 1-4).

Grãos de pólen em mônades, pequenos (tabela 1), apolares, esféricos, pantoporados com exina metarreticulada, com retículos simplescolumelados (figuras 1-3, 8-10, tabelas 3-4). Poros circulares, distribuídos dentro dos lúmens dos retículos da sexina (figura 2). As aberturas estão distribuídas por todo o grão de pólen equitativamente, sendo que Hebanthe eriantha apresenta uma média de 105 poros, enquanto que Pfaffia glomerata apresenta uma média de 73 poros, a distância entre os poros é superior em H. eriantha, mesmo apresentando um número maior de abertura, isso ocorre pela espécie apresentar grãos de pólen com diâmetros superiores (tabelas 2-3). A ornamentação dos metarretículos é formada por muros pentagonais ou hexagonais no mesmo grão de pólen (figuras 4, 10). As médias das medidas dos lúmens dos grãos de pólen analisados são superiores a $3 \mu \mathrm{m}$, assim como os muros do metarretículo que apresentam valores de médias acima de 1,00 $\mu \mathrm{m}$ (tabela 4). A exina é espessa, com sexina muito mais espessa que a camada de nexina (figuras 2,7 , tabela 4). Para a espécie $H$. eriantha tanto a espessura quanto a ornamentação da exina apresenta-se em valores superiores se comparado a P. glomerata (tabela 4). Sob MEV observamos muros sinuosos do metarretículo em $H$. eriantha (figura 5), enquanto que em $P$. glomerata 

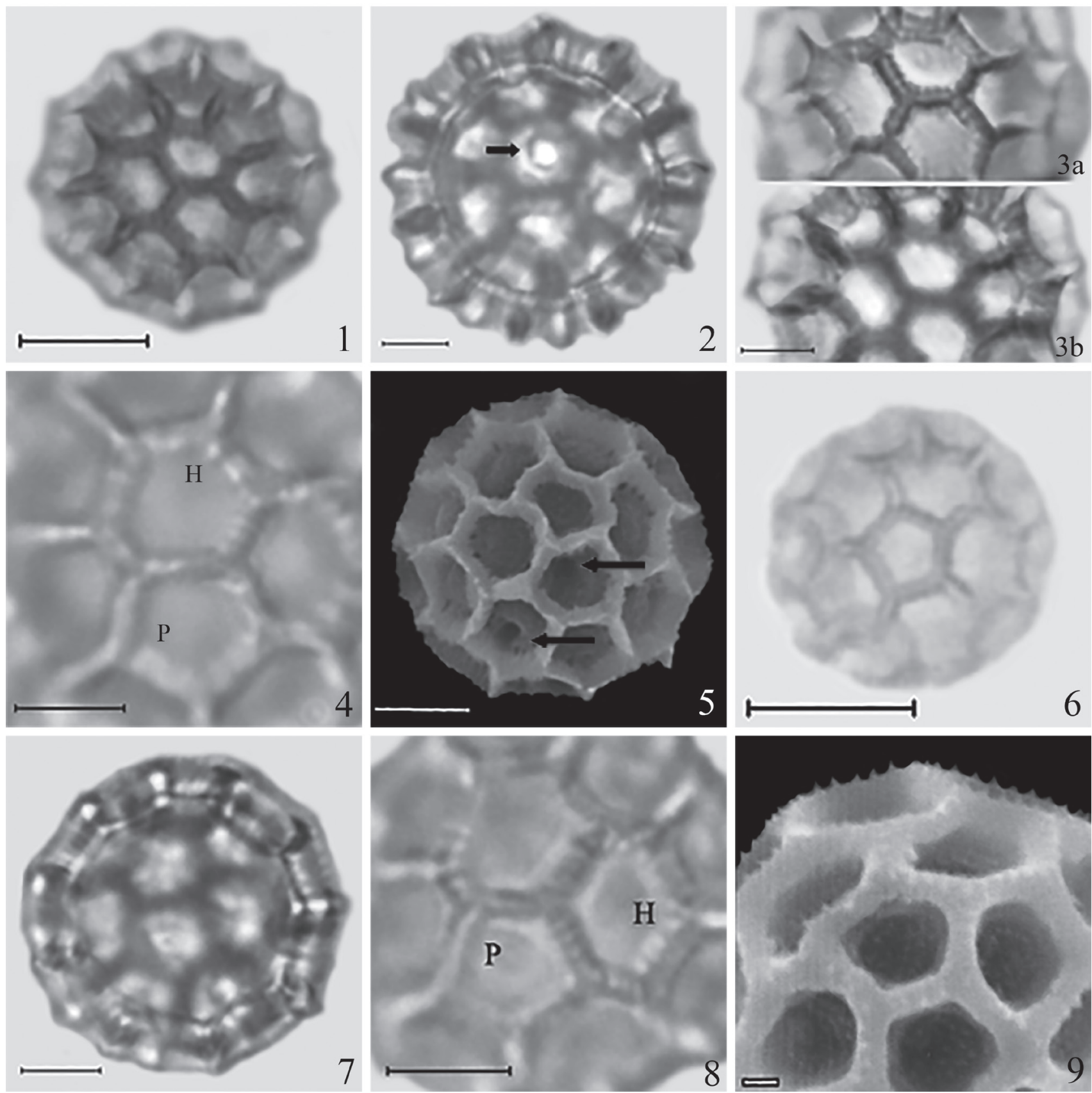

Figuras 1-9. Fotomicrografias e eletromicrografias dos grãos de pólen de espécies de Amaranthaceae nativas em fragmentos florestais remanescentes da Região Noroeste do Estado de São Paulo. 1-5. Hebanthe eriantha (Poir.) Pedersen. 1. Vista geral. 2.Corte óptico, detalhe na abertura. 3. L.O. em foco alto e foco baixo. 4. Detalhe da ornamentação, P: pentágonos do metarretículo, H: hexágonos do metarretículo. 5. Vista geral (MEV), detalhe abertura (setas). 6-9. Pfaffia glomerata (Spreng.) Pedersen. 6. Vista geral. 7. Corte óptico. 8. Detalhe da ornamentação, P: pentágonos do metarretículo, h: hexágonos do metarretículo. 9. Vista geral (MEV), detalhe grânulos nos lúmens do metarretículo (MEV). Barras = 1 $\mu \mathrm{m}(9) ; 5 \mu \mathrm{m}(2-5,7$, 8); $10 \mu \mathrm{m}(1,6)$.

Figures 1-9. Light and scanning electron micrographs of pollen grains of Amaranthaceae from native forest fragments in the northwest region of São Paulo State. 1-5. Hebanthe eriantha (Poir.) Pedersen. 1. General view. 2. Optical section, detail of aperture. 3. L.O. in high and low focus. 4. Detail of the ornamentation, P: pentagons of the metareticulum, H: hexagons of the metareticulum. 5. General view (SEM), detail of aperture (arrows). 6-9. Pfaffia glomerata(Spreng.) Pedersen. 6. General view. 7. Optical section. 8. Detail of the ornamentation, P: Pentagons of the metareticulum, H: hexagons of the metareticulum. 9. General view (SEM), detail of granula in the metareticulum lumina (SEM). Bars $=1 \mu \mathrm{m}(9) ; 5 \mu \mathrm{m}(2-5$, $7,8) ; 10 \mu \mathrm{m}(1,6)$. 

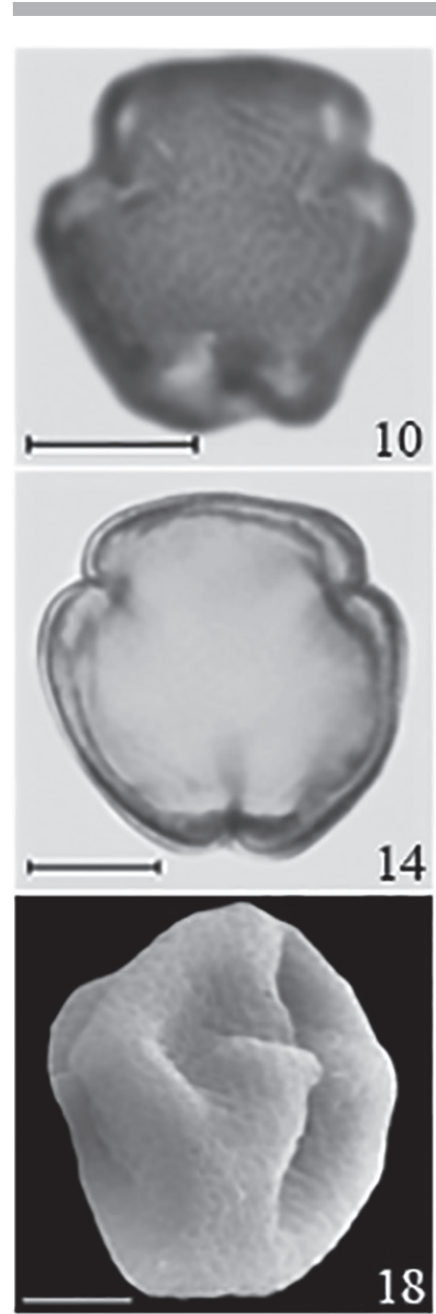

14

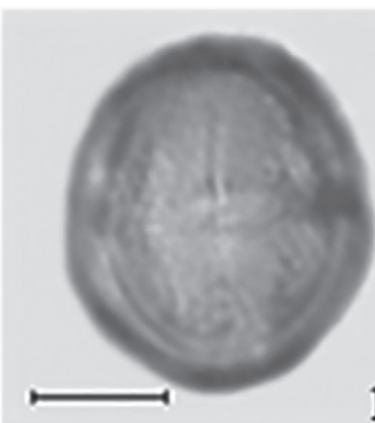

11
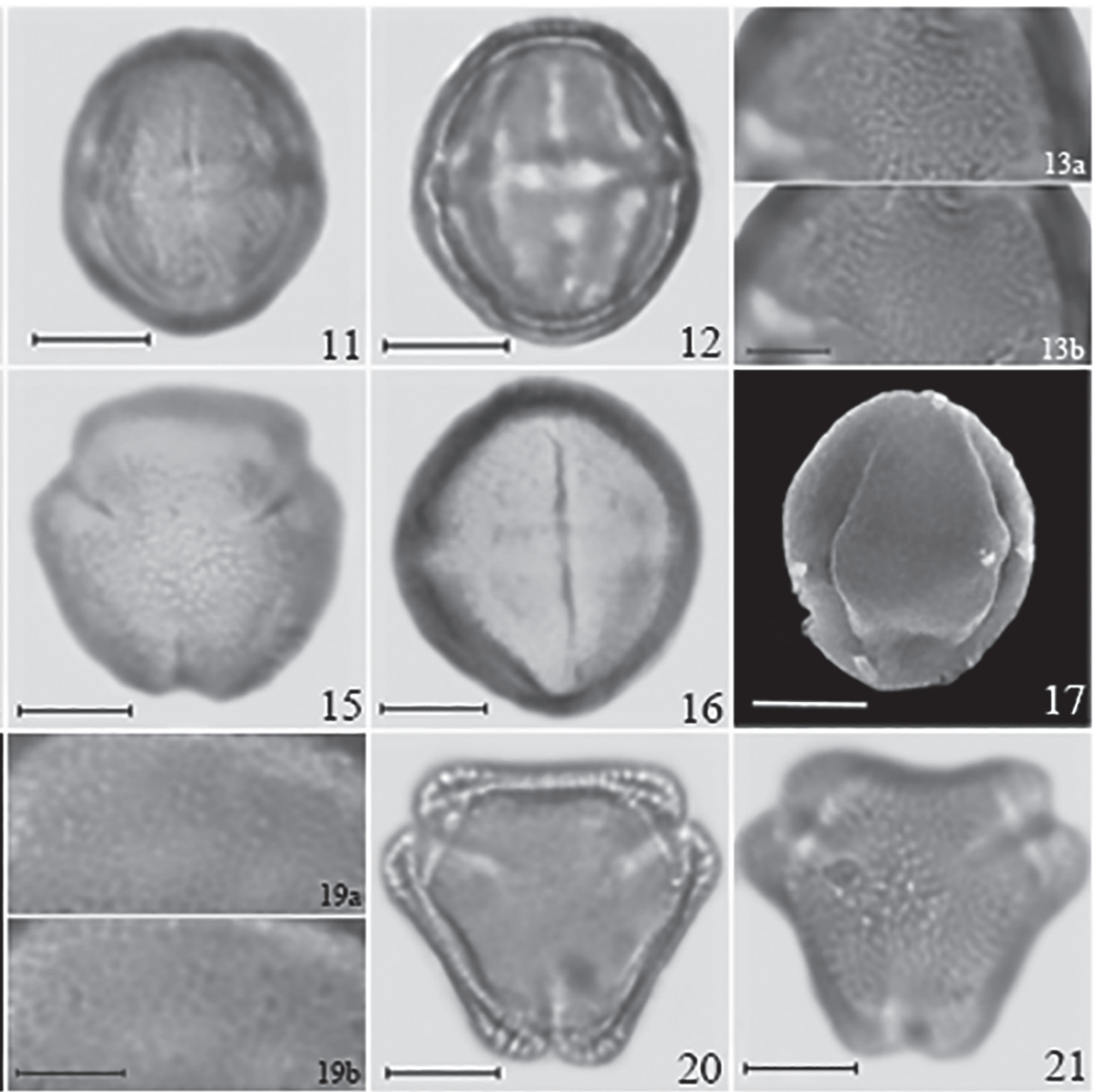

15

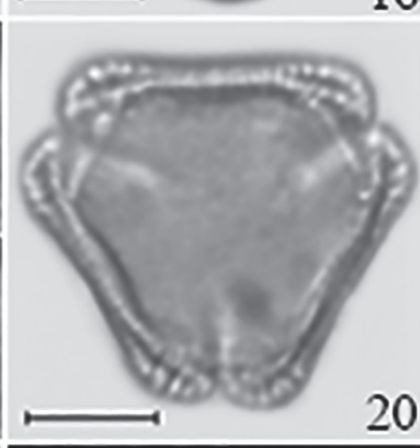

16
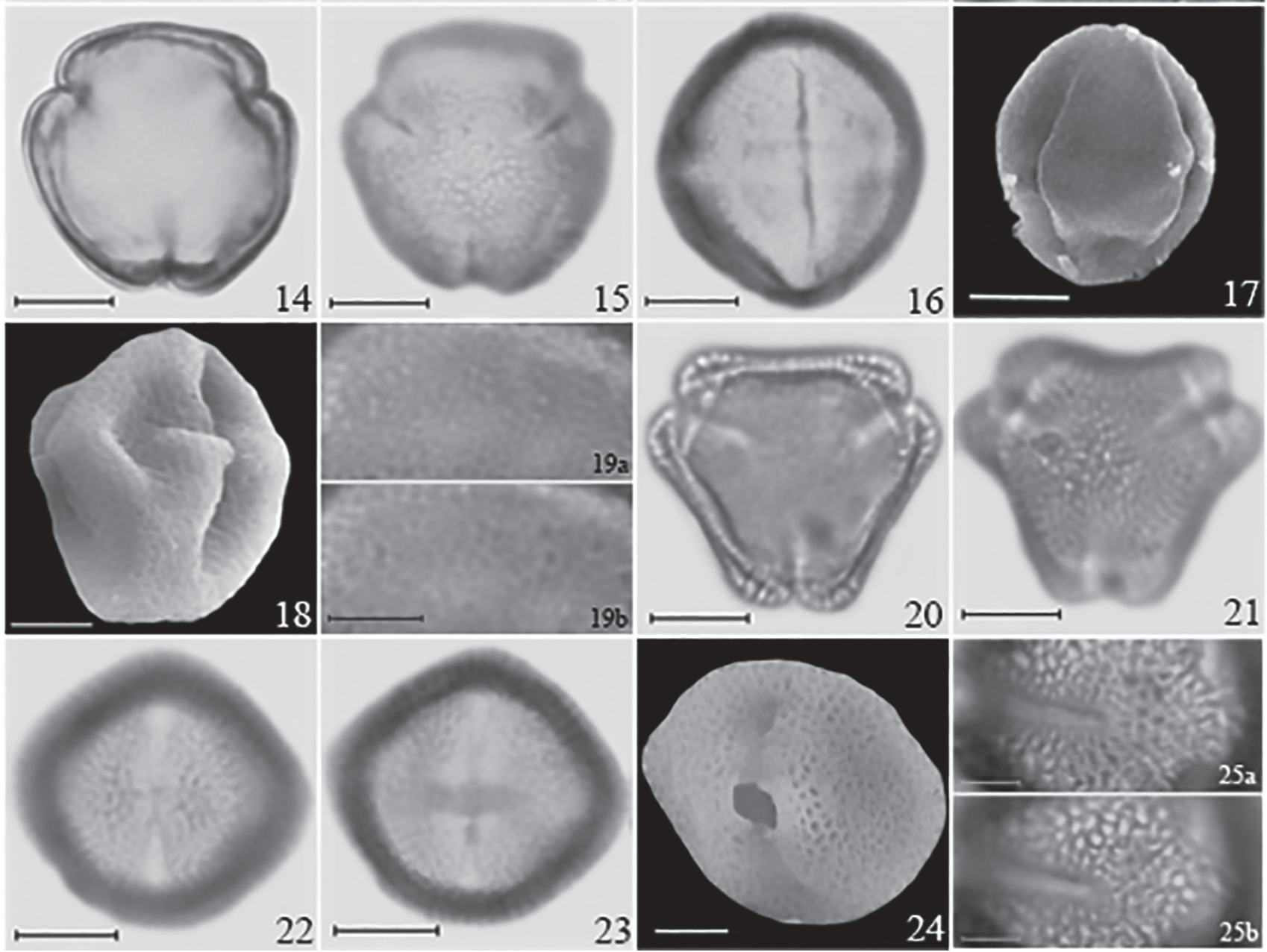

22
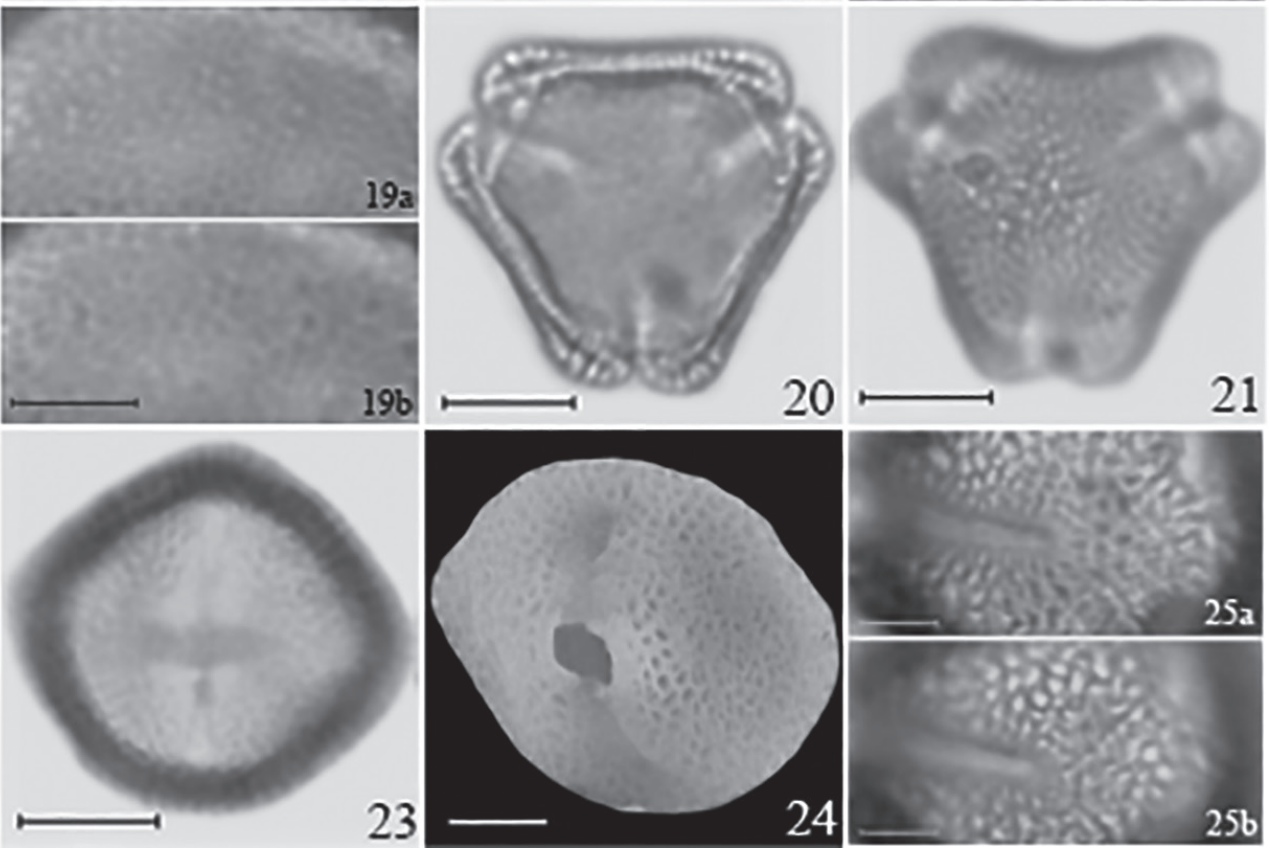

Figuras 10-25. Fotomicrografias e eletromicrografias dos grãos de pólen de espécies de Araliaceae nativas em fragmentos florestais remanescentes da Região Noroeste do Estado de São Paulo. 10-13. Aralia excelsa (Griseb.) J. Wen. 10. Ornamentação, vista polar. 11.Abertura, vista equatorial. 12. Corte óptico, vista equatorial. 13. L.O. em foco alto e foco baixo. 14-19. Dendropanax cuneatus (DC.) Decne. \& Planch. 14. Corte óptico, vista polar. 15. Ornamentação, vista polar. 16. Detalhe abertura, vista equatorial. 17. Vista geral (MEV). 18. Ornamentação (MEV). 19. L.O. em foco alto e foco baixo. 20-35. Schefflera vinosa (Cham. \& Schltdl.) Frodin \& Fiaschi. 20. Corte óptico, vista polar. 21. Ornamentação, vista polar. 22. Abertura, vista equatorial. 23. Endoabertura, vista equatorial. 24. Abertura (MEV). 25. L.O. em foco alto e foco baixo.Barras $=5 \mu \mathrm{m}(13,19,25) ; 10 \mu \mathrm{m}(10-12,14-18,20-24)$.

Figures 10-25. Light and scanning electron micrographs of pollen grains of Araliaceae from native forest fragments in the northwest region of São Paulo State. 10-13. Aralia excelsa (Griseb.) J. Wen. 10. Ornamentation, polar view. 11. Aperture, equatorial view. 12. Optical section, equatorial view. 13. L.O. in high and low focus. 14-19. Dendropanax cuneatus (DC.) Decne. \& Planch. 14. Optical section, polar view. 15. Ornamentation, polar view. 16. Detail of aperture, equatorial view. 17. General view (SEM). 18. Ornamentation (SEM). 20-25. Schefflera vinosa (Cham. \& Schltdl.) Frodin \& Fiaschi. 20. Optical section, polar view. 21.Ornamentation, polar view. 22. Aperture, equatorial view. 23. Endoaperture, equatorial view. 24. Aperture (SEM). 25. L.O. in high and low focus. Bars $=5 \mu \mathrm{m}$ (13, $19,25) ; 10 \mu \mathrm{m}(10-12,14-18,20-24)$. 
Tabela 1. Caracterização morfológica dos grãos de pólen de espécies de Amaranthaceae e Araliaceae nativas em fragmentos florestais remanescentes da Região Noroeste do Estado de São Paulo. P: pequeno, M: médio, P/E: relação diâmetro polar/ diâmetro equatorial.

Table 1. Morphologic characterization of Amaranthaceae and Araliaceae pollen grains from native forest fragments in the northwest region of São Paulo State. P: small, M: medium, P/E: ratio between polar diameter and equatorial diameter.

\begin{tabular}{lcccc}
\hline Espécies & Tamanho & $\mathrm{P} / \mathrm{E}$ & Abertura & Exina \\
\hline $\begin{array}{l}\text { Hebante eriantha } \\
\text { Pfaffia glomerata }\end{array}$ & $\mathrm{P}$ & - & pantoporado & $\begin{array}{l}\text { metarreticulado } \\
\text { metarreticulado }\end{array}$ \\
& $\mathrm{P}$ & - & & \\
Aralia excelsa & $\mathrm{P}-\mathrm{M}$ & 1,16 & 3-colporado & microrreticulado \\
Dendropanax cuneatus & $\mathrm{P}-\mathrm{M}$ & 1,17 & 3-colporado & microrreticulado \\
Schefflera vinosa & $\mathrm{P}-\mathrm{M}$ & 0,91 & 3-colporado & microrreticulado \\
\hline
\end{tabular}

Tabela 2. Dados quantitativos dos grãos de pólen de espécies de Amaranthaceae e Araliaceae nativas em fragmentos florestais remanescentes da Região Noroeste do Estado de São Paulo $(n=25)$. $\left(X_{\operatorname{mim}}-X_{\max }\right.$ : faixa de variação, x: média aritmética; $\mathrm{S}_{\mathrm{x}}$ : desvio padrão da média, S: desvio padrão da amostra, IC: intervalo de confiança a $95 \%$ da média, CV: coeficiente de variabilidade).

Table 2. Quantitative data of pollen grains of Amaranthaceae and Araliaceae from native forest fragments in the northwest region of São Paulo State $(n=25)$. $\left(\mathrm{X}_{\operatorname{mim}}-\mathrm{X}_{\max }\right.$ : variation interval, $\mathrm{x}$ : mean, $\mathrm{s}_{\mathrm{x}}$ : standard error, $\mathrm{S}$ : standard deviation, IC: confidence interval in $95 \%$ of the mean; CV: coefficient of variation).

\begin{tabular}{|c|c|c|c|c|}
\hline Espécies & $\left(\mathrm{X}_{\operatorname{mim}}-\mathrm{X}_{\max }\right) \mathrm{x} \pm \mathrm{s}_{\mathrm{x}(\mu \mathrm{m})}$ & $\mathrm{S}_{(\mu \mathrm{m})}$ & $\mathrm{IC}_{(\mu \mathrm{m})}$ & $\mathrm{CV}(\%)$ \\
\hline & \multicolumn{4}{|c|}{ Diâmetro 1} \\
\hline Hebanthe eriantha & $(18,00-23,14) 21,08 \pm 0,30$ & 1,48 & $(20,45-21,72)$ & 7,04 \\
\hline \multirow[t]{2}{*}{ Pfaffia glomerata } & $(12,86-18,00) 15,53 \pm 0,18$ & 0,90 & $(15,14-15,92)$ & 5,81 \\
\hline & \multicolumn{4}{|c|}{ Diâmetro 2} \\
\hline Hebanthe eriantha & $(18,00-23,14) 21,49 \pm 0,33$ & 1,64 & $(20,79-22,20)$ & 7,63 \\
\hline \multirow[t]{2}{*}{ Pfaffia glomerata } & $(12,86-18,00) 15,63 \pm 0,29$ & 1,47 & $(15,00-16,26)$ & 9,40 \\
\hline & \multicolumn{4}{|c|}{ Diâmetro equatorial em vista polar } \\
\hline Aralia excelsa & $(20,00-25,00) 21,65 \pm 0,27$ & 1,34 & $(21,10-22,20)$ & 6,17 \\
\hline Dendropanax cuneatus & $(20,57-30,85) 26,64 \pm 0,49$ & 2,45 & $(25,59-27,68)$ & 9,19 \\
\hline \multirow[t]{2}{*}{ Schefflera vinosa } & $(23,14-28,28) 24,68 \pm 0,33$ & 1,66 & $(23,97-25,39)$ & 6,72 \\
\hline & \multicolumn{4}{|c|}{ Diâmetro polar em vista equatorial } \\
\hline Aralia excelsa & $(25,00-27,50) 26,15 \pm 0,24$ & 1,19 & $(25,66-26,64)$ & 4,56 \\
\hline Dendropanax cuneatus & $(25,71-38,57) 33,11 \pm 0,56$ & 2,81 & $(31,91-34,32)$ & 8,48 \\
\hline \multirow[t]{2}{*}{ Schefflera vinosa } & $(20,57-28,28) 25,50 \pm 0,39$ & 1,95 & $(24,67-26,34)$ & 7,66 \\
\hline & \multicolumn{4}{|c|}{ Diâmetro equatorial em vista equatorial } \\
\hline Aralia excelsa & $(20,00-23,75) 22,40 \pm 0,12$ & 0,62 & $(22,15-22,65)$ & 2,75 \\
\hline Dendropanax cuneatus & $(23,14-30,85) 28,08 \pm 0,44$ & 2,22 & $(27,13-29,02)$ & 7,90 \\
\hline Schefflera vinosa & $(25,71-30,85) 27,97 \pm 0,31$ & 1,54 & $(27,31-28,63)$ & 5,51 \\
\hline
\end{tabular}

os muros são mais retos (figura 9), nota-se ainda granulações no interior dos lúmens do metarretículo em $H$. eriantha (figura 5) e em P. glomerata (figura 9). As duas espécies analisadas de Amaranthaceae apresentam sob o metarretículo pequenos espículos, quando observadas em MEV (figuras 5, 9).

Família Araliaceae - Espécies analisadas: Aralia excelsa (Griseb.) J.Wen (figuras 10-13; tabela 1-4), 


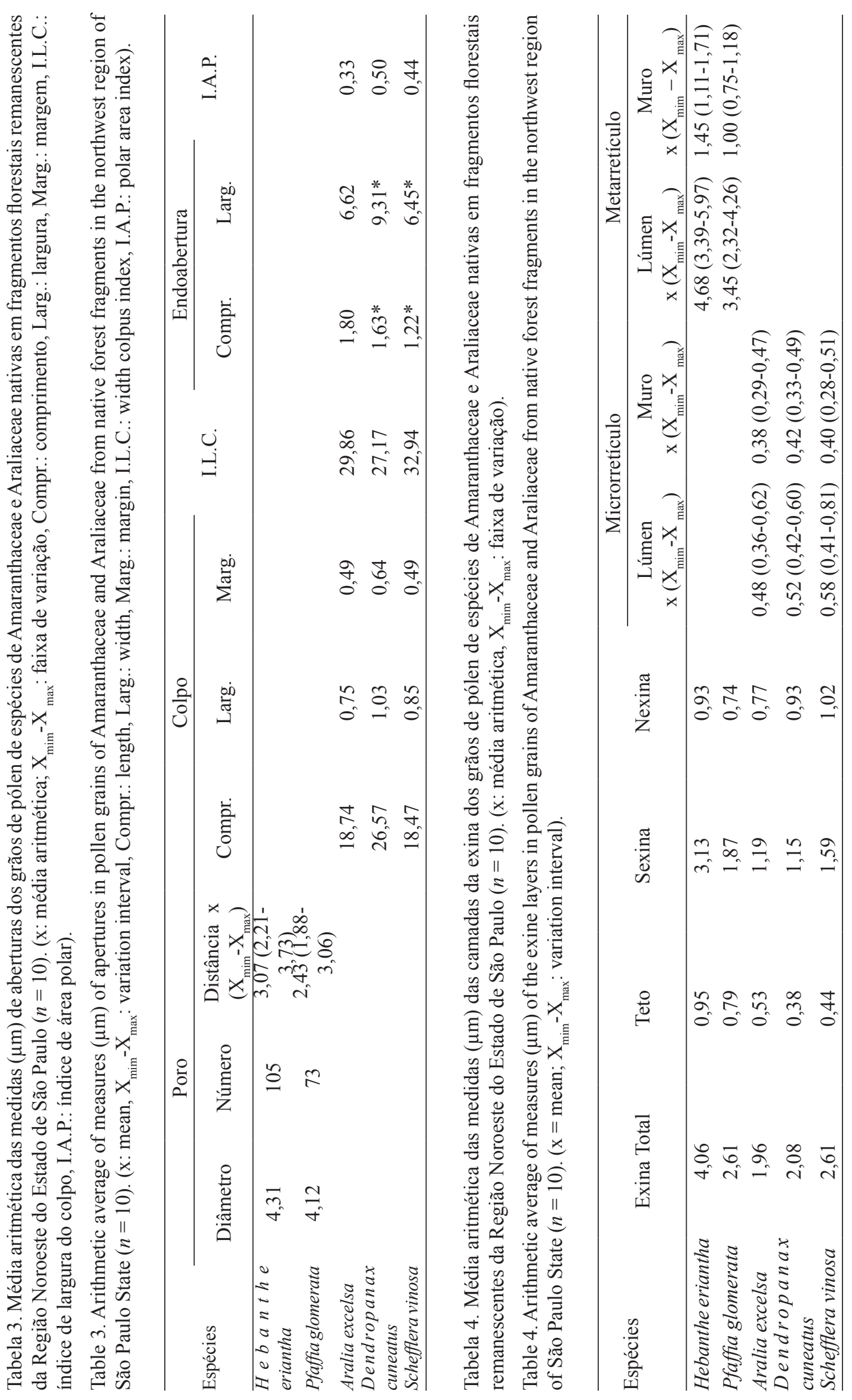


Dendropanax cuneatus (DC.) Decne. \& Planch. (figuras 14-19; tabela 1-4) e Schefflera vinosa (Cham. \& Schltdl.) Frodin \& Fiaschi (figuras 20-25; tabela $1-4)$.

Grãos de pólen em mônades, pequenos a médios (tabelas 1-2), forma oblato-esferoidal (Schefflera vinosa) a subprolato (tabela 1); isopolares com âmbito subcircular a subtriangular ( $S$. vinosa), 3-colporado, colpos longos e estreitos (tabela 3), constrictos na região mediana ( $S$. vinosa, figura 22), com extremidades afiladas (Dendropanax cuneatus, figura 15) ou arredondadas (S. vinosa, figura 21) e apresentando margem, algumas vezes com vestíbulo (S. vinosa, figura 20); endoabertura lalongada de extremidade arredondada ( $A$. excelsa, figura 11) ou afilada ( $S$. vinosa, figura 23) (tabela 3). Exina microrreticulada, sexina mais espessa que a nexina (tabela 4). A ornamentação da exina possui uma variação alta de tamanho de lúmen, variando entre $0,36 \mu \mathrm{m}$ a $0,81 \mu \mathrm{m}$, enquanto que o tamanho do muro possui uma variação menor entre as espécies, variando entre $0,29 \mu \mathrm{m}$ a $0,51 \mu \mathrm{m}$. Das três espécies de Araliaceae analisadas, $A$. excelsa é a espécie que apresenta os menores valores de exina e de microrretículos (tabela 4). Quando analisados sob microscopia eletrônica de varredura observamos microrretículos mais homogêneos em $D$. cuneatus (figura 18), já os microrretículos em $S$. vinosa são heterogêneos (figura 24).

\section{Discussão}

Segundo dados disponíveis na literatura os grãos de pólen de Amaranthaceae apresentam-se como: mônades, pequenos a médios, poliédricos, tetraédricos, dodecaedricos, subesféricos à esféricos, pantoporados com poros circulares a elípticos, variando de 4 à 250 quanto ao seu número e 12 a mais de 60 faces; sexina escabrada, espinhosa, granulada, lofada, microreticulada, reticulada com malhas grandes, metarreticulados, podendo apresentar perfurações e opérculos, formadas por muros altos, pentagonais ou hexagonais; muros simplesbaculados ou duplibaculados com reforços murais mais ou menos cônicos. Sexina podendo ser, mais ou menos espessa que a nexina (Erdtman 1952, Salgado-Labouriau 1973, Barth et al. 1976, Melhem \& Cruz, 1979, Nowicke \& Skvarla 1979, Robertson 1981, Eliasson 1988, Borsch 1995, 1998, Telleria 1995, Borsch \& Pedersen 1997, Franssen et al. 2001, Perveen \& Qaiser 2002, Evaldt et al. 2009, Bastos et al., 2010, Chin \& Lim, 2011,
Angelini et al. 2014, Magalhães-e-Silva et al., 2016, Sanchez-del-Pino et al., 2016).

Dentre os estudos citados, os trabalhos de Salgado-Labouriau (1973), Borsch (1995, 1998), Telleria (1995), Borsch \& Pedersen (1997) e Bastos et al., (2010) descrevem a morfologia polínica de espécies dos gêneros Hebanthe e Pfaffia. Para esses gêneros os grãos de pólen são descritos como esféricos, possuindo de 10-12 malhas visíveis numa face; pantoporados com poro circular dentro de cada lúmen; sexina reticulada, de malhas grandes, pentagonais ou hexagonais apresentando lados retos; muros altos com teto de superfície espiculada, com espículos dispostos geralmente em linhas, podendo possuir perfurações de forma desigual pelo grão de pólen; muros simplicolumelados ou duplicolumelados, reforços murais cônicos nos vértices das malhas.

Bastos et al. (2010) analisaram a morfologia polínica das espécies aqui descritas. As características aqui analisadas confirmam as descritas pelos autores, no entanto verificamos medidas da exina com valores ligeiramente superiores aos observados por Bastos et al. (2010). Borsch \& Pedersen (1997) e Borsh (1998), ainda comparam as diferenças entre os gêneros, estes trabalhos descrevem para o gênero Hebanthe grãos de pólen com maiores valores de diâmetros e exina mais espessa quando comparados com os das espécies de Pfaffia. Geralmente nos grãos de pólen de Hebanthe o teto da exina, próximo ao poro, apresenta grandes perfurações, fazendo com que as columelas fiquem parcialmente visíveis, o que não é observado para os grãos de pólen de Pfaffia (Borsch \& Pedersen 1997, Borsh 1998). A diferenciação entre os gêneros descrita pelos autores também foi visualizada nas espécies estudadas, principalmente quanto aos valores de diâmetros e espessura da exina.

Erdtman (1952) apresentou pela primeira vez a descrição de dois tipos polínicos para as espécies de Amaranthaceae, tipo Amaranthus L., caracterizado por apresentar grãos de pólen com elevado número de poros e espículos ou espículos vestigiais na camada de sexina, e tipo Gomphrena L. com um número menor de poros distribuídos pelos grãos de pólen, e estes dispostos em depressões situados dentro do lúmen de estruturas similares a retículos. Posteriormente, outros autores como Nowicke \& Skvarla (1979), Robertson (1981), Eliasson (1988), Telleria (1995), Perveen \& Qaiser (2002) e Sanchez-del-Pino et al. (2016) seguiram a divisão dos tipos polínicos para as espécies de Amaranthaceae considerando as subfamílias Amaranthoideae, com numerosos poros, 
distribuídos por todo o grão de pólen, não localizados em depressões da exina, ornamentação espinhosa e geralmente perfurada, e Gomphrenoideae, com grãos de pólen com um número menor de poros, localizados em depressões de muros de formas poligonais: quadrados, pentágonos ou hexágonos, separados por fileiras de columelas, de ornamentação reticulada (metarreticulada), homobrocada e simplicolumelada.

As espécies aqui analisadas apresentam morfologia polínica do tipo Gompherenoideae, apresentando metarretículos com aberturas circulares (poros) em depressões da exina, dentro dos lúmens dos retículos, separados pelos muros pentagonais e/ou hexagonais. Apesar das discretas variações na morfologia polínica de Hebanthe eriantha e Pfaffia glomerata, o caráter estenopolínico de Amaranthaceae foi observado como sugerido anteriormente por Melhem \& Cruz (1979).

Araliaceae apresenta grãos de pólen em mônades, pequenos a médios, âmbito circular, subtriangular e triangular; anguloaperturados; área polar pequena a muito pequena, oblato-esferoidais a subprolato; 3-porados, 3-colpados ou 6 colpóides, geralmente 3 -colporados, raramente 2 ou 4-colporado, colpos longos a muito longos, estreitos, de difícil visualização e mensuração, apresentando ou não margem; extremidades dos colpos arredondadas ou afiladas, podendo apresentar constrição e endocingulo; endoabertura frequentemente lalongada, lolongada e circular, podendo apresentar-se em forma de $\mathrm{H}$, de difícil visualização e mensuração devido à ornamentação da exina. Exina estriado-reticulada, espinhosa, gemada, microrreticulada, perfurado-rugulosa, reticulada homo ou heterobrocada, rugulado-reticulada, muros simpli, dupli ou multicolumelados, algumas espécies podem não apresentar teto, possuindo a camada de sexina frequentemente mais espessa que a camada de nexina (Erdtman, 1952, Chung e Huang, 1972, SalgadoLabouriau, 1973, Tseng \& Shoup, 1978, Tseng et al., 1983, Van Helvoort \& Punt, 1984, Melhem \& Bissa, 1985, Shang \& Callen, 1988, Pire, 1989, Henwood, 1991, Wen \& Nowicke, 1999, Melhem et al., 2003, Fiaschi et al., 2008, 2010).

Shang \& Callen (1988) ao estudarem os grãos de pólen de Araliaceae, descreveram três tipos polínicos baseados na forma dos grãos de pólen e cinco tipos polínicos considerando sua ornamentação. Segundo os autores, os gêneros Aralia, Dendropanax e Schefflera, possuem grãos de pólen subprolatos, e foram classificados dentro de um mesmo tipo polínico quanto à forma. Já em relação à ornamentação, Shang \& Callen (1988) agruparam as espécies de Aralia e
Dendropanax no tipo polínico para grãos de pólen com ornamentação reticulada (Aralia em um subtipo para grãos de pólen com retículos médios, lúmens entre 0,5-1,5 $\mu \mathrm{m}$, e Dendropanax no subtipo de retículos finos, com teto psilado ou lúmen inconspícuo). Para as espécies de Shefflera, Shang \& Callen (1988) não definiram a ornamentação dos grãos de pólen, mas indicaram a similaridade destes com grãos de pólen de Tupidanthus Hook.f. \& Thomson.

Aralia excelsa e Dendropanax cuneatus aqui analisadas apresentam grãos de pólen subprolatos, conforme descrito por Shang \& Callen (1988), no entanto foi observada nos grãos de pólen de Schefflera vinosa forma suboblata a oblato-esferoidal. Em relação à ornamentação dos grãos de pólen foram verificados grãos de pólen microrreticulados para as três espécies de Araliaceae analisadas, diferindo portanto do trabalho de Shang \& Callen (1988).

Os grãos de pólen de Aralia foram definidos por Wen \& Nowicke (1999) como médios, subprolatos, de âmbito subtriangular a triangular, 3-colporados com margem, endoaberturas lalongada, ornamentação estriado-reticulada, perfurada a reticulada com columelas muito curtas. Wen \& Nowicke (1999) estudaram seis espécies do gênero Aralia. A descrição realizada pelos autores para as espécies do gênero são similares aos dados aqui encontrados para $A$. excelsa, no entanto verificamos grãos de pólen pequenos, de âmbito subcircular e ornamentação microrreticulada.

Melhem \& Bissa (1985) descreveram para Dendropanax exilis (Toledo) Jung grãos de pólen médios, de âmbito triangular, prolato-esferoidais, 3-colporados, com margem e endoaberturas lalongadas e ornamentação reticulada. Os grãos de pólen de Dendropanax cuneatus aqui analisados diferem quanto ao âmbito, forma e ornamentação dos dados de Melhem \& Bissa (1985), sendo respectivamente subcirculares, subprolatos com ornamentação microrreticulada, o que pode sugerir pequenas variações morfopolínicas para as espécies do gênero. Tseng \& Shoup (1978), ao estudarem os grãos de pólen de 48 espécies de Schefflera, descreveram oito tipos polínicos com base nas variações da ornamentação. Schefflera vinosa foi descrita pelos autores dentro do tipo "digitada" com grãos de pólen esferoidais, possuindo endoaberturas no centro do colpo, sexina semitectada, reticulada, e camada de sexina mais espessa que a camada de nexina (Tseng \& Shoup 1978). Analisando as espécies de Schefflera da região Sudeste do Brasil, Fiaschi et al. (2008) propuseram dois tipos polínicos separando grãos de pólen 
reticulados (tipo 1) e regulados-reticulados (tipo 2). Já em um trabalho mais amplo, envolvendo espécies de Schefflera do Neotrópico, Fiaschi et al. (2010) ampliaram a descrição polínica, sugerindo cinco tipos polínicos para os 43 táxons analisados também com base na ornamentação dos grãos de pólen. Os dados aqui descritos para Schefflera vinosa confirmam os resultados obtidos por Tseng \& Shoup (1978), Fiaschi et al. (2008) e Fiaschi et al. (2010), observando grãos de pólen com ornamentação reticulada, heterobrocada.

As espécies de Araliaceae aqui analisadas apresentam em geral morfologia polínica muito similar, principalmente entre as espécies Aralia excelsa e Dendropanax cuneatus, no entanto para os grãos de pólen de Schefflera vinosa foram observadas diferenças quanto ao âmbito (subtriangular), a presença de vestíbulo e microrretículos com lúmens mais heterogêneos que os grãos de pólen das demais espécies.

Para as espécies aqui estudadas pode-se concluir que os grãos de pólen de Hebanthe eriantha e Pfaffia glomerata confirmam o caráter estenopolínico de Amaranthaceae, enquanto que em Araliaceae, mesmo a morfologia polínica sendo semelhante, as espécies estudadas possuem características nos seus grãos de pólen que podem ser usadas para diferenciá-las.

\section{Literatura citada}

Angelini, P., Bricchi, E., Gigante, G., Poponessi, S., Spina, A. \& Venanzoni, R. 2014. Pollen morphology of some species of Amaranthaceae s. lat. common in Italy. Flora Mediterranea 24:247-272.

APG, IV. 2016. An update of the Angiosperm Phylogeny Group classification for the orders and families of flowering plants. The Linnean Society of London, Botanical Journal of the Linnean Society.

Barth, O.M. \& Melhem, T.S. 1988. Glossário ilustrado de Palinologia. Editora da Universidade Estadual de Campinas, Campinas.

Barth, O.M., Corte-Real, S. \& Macieira, E.G. 1976. Morfologia do pólen anemófilo e alergizante no Brasil. II Polygonaceae, Amaranthaceae, Chenopodiaceae, Leguminosae, Euphorbiaceae e Myrtaceae. Rio de Janeiro. Memórias do Instituto Oswaldo Cruz 74: 191-201.

Bastos, J.R., Bauermann, S.G. \& Marchioretto, M.S. 2010. Morfologia polínica dos gêneros Hebanthe Mart. e Pfaffia Mart. nativos do Rio Grande do Sul. Revista de Iniciação Científica da ULBRA: 37-42.

Borsch, T. 1995. Three new combinations in Pfaffia (Amaranthaceae) from the New World Tropics. Novon 5: 230-233.
Borsch, T. 1998. Pollen types in the Amaranthaceae. Morphology and evolutionary significance. Grana 37: 129-142.

Borsch, T. \& Pedersen, T.M. 1997. Restoring the Generic Rank of Hebanthe Martius (Amaranthaceae). Sendtnera 4: 13-31.

Chin, E.H.S. \& Lim, A.L. 2011. Comparative pollen morphology of three Alternanthera species (Amaranthaceae). Garden's Bulletin Singapore 63: 471-483.

Chung, T.F. \& Huang, T.C. 1972. Paleoecological Study of Taipei Basin. Taiwania 17: 117-141.

Erdtman, G. 1952. Pollen morphology and plant taxonomyAngiosperms. Alquimist \& Wiksell, Stockholm.

Erdtman, G. 1960.The acetolysis method. A revised description. Svensk Botanisk Tidskrift 54: 561-564.

Eliasson, U.H. 1988. Floral morphology and taxonomic relations among the genera of Amaranthaceae in the New World and the Hawaiian Islands. Botanical Journal of the Linnean Society 96: 235-283.

Evaldt, A. C.P., Bauermann, S.G. \& Fuchs, S.C.B. 2009. Grãos de pólen e esporos do Vale do Rio Caí, nordeste do Rio Grande do Sul, Brasil: descrições morfológicas e implicações paleoecológicas. Gaea - Jounal of Geoscience 5: 86-106.

Faegri, G. \& Iversen, J. 1966. Textbook of modern pollen analysis. 2 ed. Scandinavian University Books, Copenhagen.

Fiaschi, P., Cruz-Barros, M.A.V. \& Correa, A.M.S. 2008. Estudo palinotaxonômico de espécies de Schefflera (Araliaceae) da região sudeste do Brasil. Rodriguésia 59: 873-886.

Fiaschi, P., Santos, F.A.R., Westbrook, E. \& Plunkett, G.M. 2010. Taxonomic significance of pollen morphology in Neotropical Schefflera (Araliaceae). Plant Diversity and Evolution 128: 297-323.

Fiaschi, P. 2015. Araliaceae in Lista de Espécies da Flora do Brasil. Jardim Botânico do Rio de Janeiro. Disponível em http://floradobrasil.jbrj.gov.br/jabot/floradobrasil/ FB52 (acesso 19-II-2017).

Franssen, A.S., Skinner, D.Z., Al-Khatib, K. \& Horak, M.J. 2001. Pollen morphological differences in Amaranthus species and interspecific hybrids. Weed Science 49: 732-737.

Gasparino, E.C., Cruz-Barros, M.A.V. \& Chautems, A. 2013. Pollen morphology in Brazilian species of Codonanthe (Mart.) Hanst. and Nematanthus Schrader (Gesneriaceae). Grana 52: 285-274.

Henwood, M.J. 1991. Pollen morphology of Polyscias (Araliaceae): The Malesian and Australian species. Grana 30: 559-576.

Magalhães-e-Silva, F.H., Santos, F.A.R. \& Lima, L.C.L. 2016. Flora polínica das caatingas: Estação Biológica de Canudos (Canudos, Bahia, Brasil), Feira de Santana, Bahia. 
Maury, C. 2002. Avaliação e identificação de áreas e ações prioritárias para a conservção, utilização sistentável e repartição dos benefícios da biodiversidade nos biomas brasileiros. Ministério do Meio Ambiente/Secretaria de Biodiversidade e Floresta. Brasília.

Marchioretto, M.S., Senna, L. \& Siqueira, J.C. 2015. Amaranthaceae in Lista de Espécies da Flora do Brasil. Jardim Botânico do Rio de Janeiro. Disponível em http://floradobrasil.jbrj.gov.br/jabot/floradobrasil/FB42 (acesso 19-II-2017).

Melhem, T.S. \& Bissa, W.N. 1985. Flora polínica da reserve do parque Estadual das Fontes de Ipiranga, São Paulo, Brasil. Famílias 35-Dilleniaceae, 38-Theaceae e 46-Bombacaceae. Hoehnea 12: 5-9.

Melhem, T.S. \& Cruz, M.A.V. 1979. Grãos de pólen de plantas alergógeneas: Amaranthaceae e Chenopodiaceae. Hohenea 8: 47-56.

Melhem, T.S. \& Matos, M.E.R. 1972. Variabilidade de forma dos grãos de pólen de Eriope crassipes Benth.Labiatae. Hoehnea 2: 1-10.

Melhem, T.S., Cruz-Barros, M.A.V., Corrêa, M.A.S., Makino-Watanabe, H., Silvestre-Capelato, M.S.F. \& Gonçalves-Esteves, V.L. 2003. Variabilidade polínica em plantas de Campos do Jordão (São Paulo, Brasil). Boletim do Instituto de Botânica de São Paulo, 16: 1-104.

Necchi, O. Jr. 2012. Fauna e Flora de fragmentos florestais remanescentes da região Noroeste do Estado de São Paulo. Holos Editora, Ribeirão Preto.

Nowicke, J.W. \& Skvarla, J.J. 1979. Pollen Morphology: The Potential Influence In Higher Order Systematics. Annals of the Missouri Botanical Garden 66: 633-700.

Perveen, A \& Qaiser, M. 2002. Pollen Flora of Pakistan XVIII. Amaranthaceae. Pakistan Journal Botany, 34: 375-383.

Pire, S.M. 1989. Morfologia polinica de las Araliaceae de Argentina. Bonplandia 6: 133-150.

Punt, W., Hoen, P.P., Blackmore, S., Nilsson, S. \& Le Thomas, A. 2007.Glossary of pollen and spore terminology. Review of Paleobotany and Palynology. 143: 1-81.
Ranga, N.T., Rezende, A.A., Cavasan, O., Toniato, M.T.Z., Cielo-Filho, R. \& Stranghetti, V. 2012. Caracterização florística de remanescentes de vegetacção nativa da região noroeste do Estado de São Paulo. In: O. Jr. Necchi (ed). Fauna e Flora de fragmentos florestais remanescentes da região Noroeste do Estado de São Paulo. Holos Editora, Ribeirão Preto, pp. 105-135.

Robertson, K.R. 1981. The Genera of Amaranthaceae in the Southeastern United States, Journal of the Arnold Arboretum 62: 267-313.

Salgado-Labouriau, M.L. 1973.Contribuição à Palinologia dos Cerrados. Academia Brasileira de Ciências, Rio de Janeiro.

Sánchez-del Pino, I. Fuentes-Soriano, S. SolisFernández, K.Z., Pool, R. \& Alfaro, R. 2016. The metareticulate pollen morphology of Alternanthera Forssk. (Gomphrenoideae, Amaranthaceae) and its taxonomic implications. Grana 55: 1-25.

Shang, C. \& Callen, D. 1988. Pollen morphology of the family Araliaceae in China. Bulletin of Botanical Research 8: 13-48.

Tellería, M. C. 1995. El Polen de las Mieles del Noroeste de la Provincia de Buenos Aires, Argentina. Darwiniana 33: 347-364.

Tseng, C.C. \& Shoup, J.R. 1978. Pollen morphology os Schefflera (Araliaceae). American Jounal of Botany 65: 384-394.

Tseng, C.C., Shoup, J.R., Chuang, T. \& Hsieh, W.C. 1983. Pollen morphology of Acanthopanax (Araliaceae). Grana 22: 11-17.

Van Helvoort, H.A.M. \& Punt, W. 1984. The Northwest European Pollen Flora, 29 - Araliaceae. Review of Palaeobotany and Palynology 42:1-5.

Viana, V.M. 1995. Conservação da biodiversidade de fragmentos de florestas tropicais em paisagens intensamente cultivadas. In: Abordagens interdisciplinares para a Conservação da Biodiversidade e Dinâmica do Uso da Terra no Novo Mundo. Gainesville: Conservation International do Brasil/ Universidade Federal de Minas Gerais/University of Florida, pp. 135-154.

Wen, J. \& Nowicke, J.W. 1999. Pollen ultrastructure of Panax (The Ginseng genus, Araliaceae), an eastern Asian and eastern north American disjunct genus. American Jounal of Botany 86: 1624-1636. 